\section{Absent internal jugular vein: Another case for ultrasound guided vascular access}

Sir,

We describe a case of absence of right internal jugular vein (IJV), which is a rare form of anatomical variation of the IJV. A 65-year-old male patient was admitted to the intensive care unit (ICU) with the complaints of fever and hypotension. The ICU resident placed a central venous catheter (CVC) in left IJV as he was unable to visualize the right IJV on ultrasound. On the $10^{\text {th }}$ ICU day, the CVC insertion site was found to be inflamed, and it was decided to resite the catheter on the right side.

The right side of the neck was examined carefully, and no evidence of scarring suggesting previous surgery or cannulation was seen. The ICU consultant scanned the right side of the neck carefully using a portable ultrasound (US) machine (IMAGIC Agile, Kontron Medical, WA, USA) with a linear, high frequency transducer (7.5-12 MHz). Care was taken to apply minimal pressure on the probe to prevent collapse of the IJV. Imaging showed a single pulsatile vessel, which was non compressible suggestive of the carotid artery with

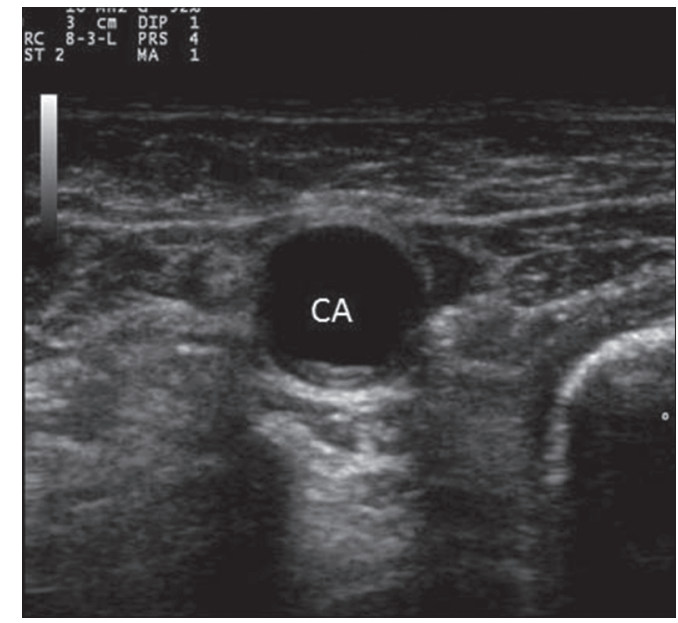

Figure I: Ultrasound image of the right side of the neck showing absence of internal jugular vein. CA: Carotid artery no evidence of the IJV [Figure 1]. Doppler confirmed the characteristic pulsatile blood flow in the carotid artery. The 2D US imaging on the left side showed normal anatomy with good size IJV [Figure 2]. Subsequently, the CVC was placed in the right axillary vein under real-time US guidance in a single attempt.

Landmark guided central venous cannulation is based on the premise of normal anatomy of the vein and its adjoining structures. In a study on long-term hemodialysis patients, the incidence of significant US findings such as total occlusion, nonocclusive thrombus and stenosis was seen to be as high as $35 \% .{ }^{[1]}$ Agenesis or hypoplasia of the central veins though rare is another probable cause of failed cannulation in patients where US guidance is not used. In our patient, the possible absence of the vein can be explained by IJV agenesis, which represents a form of truncular venous malformation due to a developmental arrest during the later stages of embryonic development, which could lead to either aplasia or hypoplasia of the vein. ${ }^{[2]}$ Denys and Uretsky studied 200 patients undergoing IJV cannulation under US guidance and found that in $2.5 \%$ of the patients, the IJV was not visualized. ${ }^{[3]}$ Absence of the right sided IJV has also been reported in a 12-year-old boy during US evaluation prior to attempted cannulation. ${ }^{[4]}$ In another report, IJV agenesis was discovered during neck dissection. Patients who require removal of IJV due to disease infiltration may have potentially life-threatening complication of cerebral edema if the other IJV is aplastic. ${ }^{[5]}$

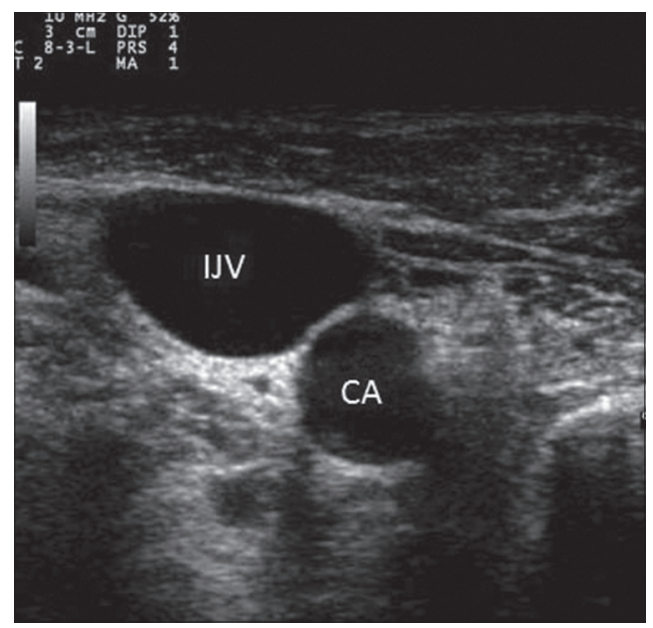

Figure 2: Ultrasound image of the left side of the neck showing normal anatomy. CA: Carotid artery; IJV: Internal jugular vein 
In developing countries, landmark based technique is commonly practiced as an US machine may not be available in all centers. Central venous cannulation using the landmark technique in a patient with absent IJV may be associated with a higher incidence of complications such as arterial puncture and pneumothorax. ${ }^{[6]}$ Therefore, repeated attempts to cannulate the IJV should be avoided in patients keeping in mind the possibility of anatomical variations of IJV. This case reiterates the use of US guided vascular access to prevent complications, which can arise due to the absence of a vein or other venous anomalies. In addition, it has been shown that US-guided CVC placement technique is easy to learn even by novices and any previous CVC and/or US experience allows a better technique and shorter total time to placement. ${ }^{[7]}$ It would be a good general practice to use US for both assessment of anatomy prior to venipuncture and real-time visualization during insertion of CVC to increase the success rate and prevent complications.

\section{Vimi Rewari, Ravindran Chandran, Rashmi Ramachandran, Anjan Trikha Department of Anaesthesiology, All India Institute of} Medical Sciences, New Delhi, India

Correspondence : Prof. V. Rewari, Department of Anaesthesiology, All India Institute of Medical Sciences, Ansari Nagar, New Delhi - 110 029, India.

E-mail: vimirewari@gmail.com

\section{References}

1. Forauer AR, Glockner JF. Importance of US findings in access planning during jugular vein hemodialysis catheter placements. J Vasc Interv Radiol 2000;11:233-8.

2. Lee AB, Laredo J, Neville R. Embryological background of truncular venous malformation in the extracranial venous pathways as the cause of chronic cerebro spinal venous insufficiency. Int Angiol 2010;29:95-108.

3. Denys BG, Uretsky BF. Anatomical variations of internal jugular vein location: Impact on central venous access. Crit Care Med 1991;19:1516-9.

4. Miller BR. Absence of a right internal jugular vein detected by ultrasound imaging. Paediatr Anaesth 2011;21:91.

5. Majeed TA, Deshpande RK, Upadhaya S, Deshmukh SA. Agenesis of internal jugular vein a cause for concern. Indian J Surg Oncol 2010;1:341-2.

6. Scott DH. 'In the country of the blind, the one-eyed man is king', Erasmus (1466-1536). Br J Anaesth 1999;82:820-1.

7. Nguyen BV, Prat G, Vincent JL, Nowak E, Bizien N, Tonnelier JM, et al. Determination of the learning curve for ultrasound-guided jugular central venous catheter placement. Intensive Care Med 2014;40:66-73.

\begin{tabular}{|l|l|}
\hline \multicolumn{2}{|c|}{ Access this article online } \\
\hline Quick Response Code: & Website: \\
\hline
\end{tabular}

